\title{
Leveraging Uncertainty Estimates to Improve Segmentation Performance in Cardiac MR
}

\author{
Tewodros Weldebirhan Arega $\left({ }^{凶}\right)$, Stéphanie Bricq, and Fabrice Meriaudeau \\ ImViA Laboratory, Université Bourgogne Franche-Comté, Dijon, France \\ ${ }_{\text {tewdrosw }}$ [at] gmail.com
}

\begin{abstract}
In medical image segmentation, several studies have used Bayesian neural networks to segment and quantify the uncertainty of the images. These studies show that there might be an increased epistemic uncertainty in areas where there are semantically and visually challenging pixels. The uncertain areas of the image can be of a great interest as they can possibly indicate the regions of incorrect segmentation. To leverage the uncertainty information, we propose a segmentation model that incorporates the uncertainty into its learning process. Firstly, we generate the uncertainty estimate (sample variance) using Monte-Carlo dropout during training. Then we incorporate it into the loss function to improve the segmentation accuracy and probability calibration. The proposed method is validated on the publicly available EMIDEC MICCAI 2020 dataset that mainly focuses on segmentation of healthy and infarcted myocardium. Our method achieves the state of the art results outperforming the top ranked methods of the challenge. The experimental results show that adding the uncertainty information to the loss function improves the segmentation results by enhancing the geometrical and clinical segmentation metrics of both the scar and myocardium. These improvements are particularly significant at the visually challenging and difficult images which have higher epistemic uncertainty. The proposed system also produces more calibrated probabilities.
\end{abstract}

Keywords: Cardiac MRI Segmentation · Myocardial scar · Uncertainty - Bayesian deep learning

\section{Introduction}

Cardiac magnetic resonance (CMR) is a set of magnetic resonance imaging (MRI) used to provide anatomical and functional information of the heart. Late Gadolinium Enhancement (LGE), sometimes called delayed-enhancement MRI, is one type of CMR which is gold standard for the quantification of myocardial infarction. Myocardial infarction, also called heart attack, is the interruption of coronary blood supply to certain myocardial area which leads to irreversible death of myocardial tissue [11]. No-reflow phenomenon is an incident that usually appears in a proportion of patients with acute myocardial infarction following re-perfusion therapy of an occluded coronary artery [1]. 
Recently, deep learning based semi-automatic and fully-automatic methods have been proposed to segment myocardial scar (infarction) from LGE images. Zabihollahy et al. [22] used manual segmentation for myocardium and 2D Fully Convolutional Network to segment scar from the myocardium. Zhang [23], Ma [14] and Girum et al. [8] used a two stage cascaded segmentation framework to automatically segment myocardial scar and tested their method on EMIDEC dataset. In the first stage, Zhang [23] used a 2D nnUNet [9] to get a coarse segmentation. In the second stage, a 3D nnUNet is utilized to further refine the segmentation result. Ma [14] used a 2D nnUNet [9] to first segment the whole heart as region of interest (ROI) and then utilized a second 2D nnUNet to segment the myocardial infarction from the ROI. Arega et al. [2] also used a cascaded framework of three networks to automatically segment scar from multi-sequence CMR. The main problem with these cascaded methods is that they can be time consuming and computationally expensive.

Bayesian deep learning have been used in segmentation task to provide a prediction as well as quantify the uncertainty associated with each prediction. Recently, several studies have employed Monte Carlo Dropout to estimate uncertainty for medical image segmentation [15-17,19,20]. Monte Carlo (MC) dropout is an uncertainty estimation method proposed by Gal and Ghahramani [7]. It is done by training a network with dropout and taking the Monte Carlo samples of the prediction using dropout at test time. Nair et al. [16] explored MC dropout based uncertainty estimates for multiple sclerosis lesion detection and segmentation. They improved the segmentation results by filtering and excluding the most uncertain voxels. Similarly, Sander et al. [20] applied MC Dropout based method for cardiac MRI segmentation and showed that the uncertainty maps are close to the reported segmentation errors and they improved the segmentation results by correcting the uncertain pixels. These previous studies $[10,15,16,19,20]$ mostly focused on the correlations between predictive uncertainty and the segmentation accuracy and how the uncertainty metrics can be used to improve the segmentation by filtering the most uncertain predictions. However, these methods did not leverage the uncertainty information during training to enhance the segmentation result.

In this paper, we proposed a segmentation model that generates uncertainty estimates during training using MC-dropout. Then it leverages these uncertainty estimates to improve the segmentation results by incorporating them to the loss function. Uncertainty information can possibly indicate the regions of incorrect segmentation $[20,21]$. We hypothesized that by incorporating this information as part of the learning process, it can help the network to improve the segmentation results by correcting the segmentation errors that have high epistemic uncertainty. The proposed method was evaluated on the publicly available EMIDEC MICCAI 2020 dataset [13]. It achieved the state of the art results outperforming the top ranked methods of the challenge. The experimental results showed that the uncertainty information was indeed beneficial in enhancing the segmentation performance. We also observed that the improvements were more significant at the semantically and visually challenging images which have higher epistemic 
uncertainty. Assessing the probability calibration, we showed that the proposed method produced more calibrated probabilities than the baseline method.

\section{Materials and Methods}

\subsection{Dataset}

The Automatic Evaluation of Myocardial Infarction from Delayed-Enhancement Cardiac MRI challenge (EMIDEC) ${ }^{1}$ is a MICCAI 2020 challenge that focuses on cardiac MRI segmentation. The dataset consists of LGE images of 100 patients for training. From these cases, 67 are pathological cases and the remaining 33 are normal cases. The testing set includes 50 patients in which 33 are pathological and 17 are normal cases. Each case has 5 to 10 short-axis slices covering the left ventricle from base to apex with the following characteristics: slice thickness of $8 \mathrm{~mm}$, distance between slices of $10 \mathrm{~mm}$ and spatial resolution ranging from $1.25 \times 1.25 \mathrm{~mm}^{2}$ to $2 \times 2 \mathrm{~mm}^{2}$ [13]. As a pre-processing step, we normalized the intensity of every patient image to have zero-mean and unit-variance and we resampled all the volumes to have a voxel spacing of $1.458 \mathrm{~mm} \times 1.458 \mathrm{~mm} \times$ $10.0 \mathrm{~mm}$.

\subsection{Methods}

Various Bayesian deep learning methods are used to estimate uncertainties in images. Among the most widely used Bayesian deep learning methods in medical images is Monte-Carlo dropout (MC-dropout). In MC-dropout, a network with dropout is trained, then during testing the network is sampled $\mathrm{N}$ times in order to get $\mathrm{N}$ segmentation samples. From these $\mathrm{N}$ segmentation samples, the uncertainty measure (sample variance) is computed. In our method, we used MC-dropout during training in order to get the uncertainty estimates. During training, the model is sampled $\mathrm{N}$ times and the mean of these samples is used as the final segmentation as can be seen from Fig. 1. The uncertainty metric is computed from the N Monte-Carlo dropout samples. It can be calculated per pixel or per structure [17]. In this work, we used the pixel-wise uncertainty and image-level uncertainty. Pixel-wise uncertainty is computed per pixel. Sample variance is one of the pixel-wise uncertainty measures. It is calculated as the variance of the $N$ Monte-Carlo prediction samples of a pixel. Each pixel $i$ has $N$ sigmoid predictions $\left(y_{i, 1} \ldots y_{i, N}\right)$. From these predictions, the mean $\mu_{i}$ is computed (Eq. 1). In Eq. 2, $\sigma_{i}^{2}$ is the sample variance of each pixel $i$ of the image [16]. In order to compute the image-level uncertainty, the per-pixel uncertainty is averaged over all pixels of the image as shown in Eq. 4. In this equation, $I$ is the total number of pixels of the image.

$$
\mu_{i}=\frac{1}{N} \sum_{n}\left(y_{i, n}\right)
$$

\footnotetext{
${ }^{1}$ http://emidec.com/
} 


$$
\sigma_{i}^{2}=\frac{1}{N} \sum_{n}\left(y_{i, n}-\mu_{i}\right)^{2}
$$

As stated by [20] and [21], uncertainty information indicates potential missegmentations and the most uncertain part of the segmentation results cover regions of incorrect segmentations. In order to leverage this uncertainty information, we proposed to include it as part of the loss function so that the network will learn to correct the possible mis-segmentations. Hence, the total loss is computed as a sum of the segmentation loss and uncertainty loss as can be seen from Fig. 1. The segmentation loss is the weighted average of cross-entropy (CE) loss and Dice loss (Eq. 3). For the uncertainty loss, we first computed the image level uncertainty (Eq. 4). Then, it is added to the segmentation loss with a hyperparameter value alpha $(\alpha)$ that controls the contribution of the uncertainty loss to the total loss (Eq. 5).

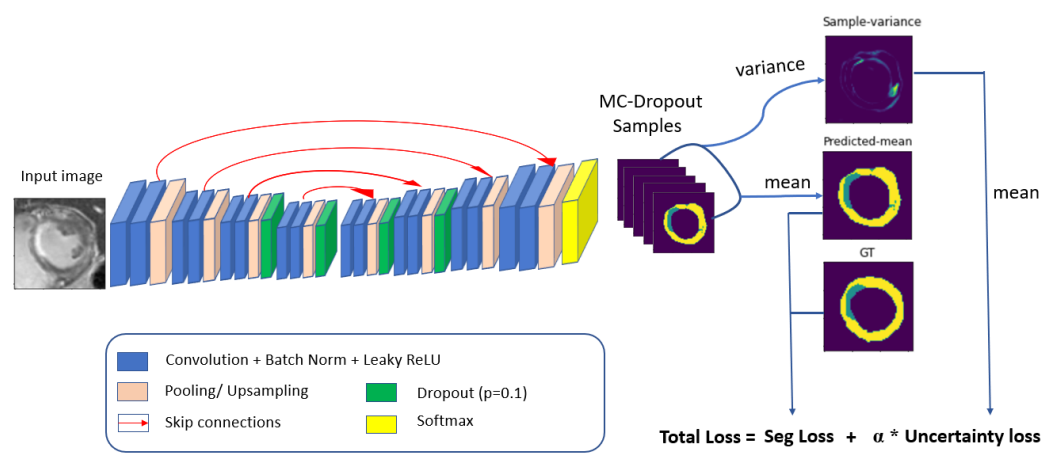

Fig. 1. The proposed method

$$
\begin{gathered}
L_{\text {Seg }}=\lambda_{\text {Dice }} L_{\text {Dice }}+\lambda_{C E} L_{C E} \\
L_{\text {Uncertainty }}=\frac{1}{I} \sum_{i}\left(\sigma_{i}^{2}\right) \\
L_{\text {Total }}=L_{\text {Seg }}+\alpha \times L_{\text {Uncertainty }}
\end{gathered}
$$

For the segmentation network, we used a 3D UNet [9] architecture with dropout placed at the middle layers of the network (Fig. 1) as suggested by the literatures $[4,6,12,17]$. The dropout rate was set at 0.1 . The UNet's encoder and decoder consists of 8 convolutional layers where each convolution is followed by batch normalization and Leaky ReLU (negative slope of 0.01) activation function. 


\subsection{Training}

The weights of the segmentation network are optimized using Stochastic gradient descent (SGD) with nesterov momentum $(\mu=0.99)$ with an initial learning rate of 0.01 . The mini-batch size was 5 and the model was trained for 1000 epochs on a five-fold cross validation scheme. For the segmentation loss, we set a weighting factor of 1.0 for Dice loss and 1.0 for CE loss as they provided the best results. In order to generate the segmentation uncertainty (sample variance), we used 5 Monte Carlo samples (the $N$ value in Eq. 1). The weighting factor $(\alpha)$ for the uncertainty loss (in Eq. 5) is empirically selected to be 3.0 after experimenting with different weighting factors. The training was done on NVIDIA Tesla V100 GPUs using Pytorch deep learning framework based on nnU-Net implementation [9].

\section{Results and Discussion}

To evaluate the segmentation results, we used geometrical metrics such as Dice coefficient (DSC) and Hausdorff distance (HD). In addition, we computed clinical metrics which are commonly used in cardiac clinical practice. These include the average volume error (VD) of the left ventricular myocardium (in $\mathrm{cm}^{3}$ ), the volume (in $\mathrm{cm}^{3}$ ) and percentage (PD) of infarction and no-reflow [13].

To measure probability calibration of the models, we used Brier score (BS). Brier score measures how close the predicted segmentation probabilities are to their corresponding ground truth probabilities (one-hot encoding of each classes) by computing the mean square error of the two probabilities [17]. To compare image level uncertainties among the segmentation results, we utilized Dice agreement within MC samples (DiceWithinSamples) $[17,19]$. It is the average Dice score of the mean predicted segmentation $\left(S_{\text {mean }}\right)$ and the individual $N$ MC prediction samples as shown in Eq. 6. Note that Dice WithinSamples is inversely related to uncertainty.

$$
\text { Dice }_{\text {WithinSamples }}=\frac{1}{N} \sum_{n} \operatorname{Dice}\left(S_{\text {mean }}, S_{n}\right)
$$

\subsection{Ablation Study}

To evaluate the effect of adding uncertainty information to the segmentation loss, we compared the model that uses only segmentation loss which is called baseline with the model that uses combined loss of segmentation loss and uncertainty loss which is referred as proposed. Both networks have the same architecture and the comparison is done on the test dataset. For the ablation study, most of the comparisons are done on the main two classes that are healthy myocardium and infarction. The comparison on all the three classes can be found in the supplementary material.

As can be seen from the table 1, the addition of uncertainty information into the segmentation loss enhanced the segmentation accuracy. It increased the 
DSC of scar (infarction) by $3 \%$ and that of myocardium by around $0.2 \%$. It also improved the HD and the average volume error of both scar and myocardium. The segmentation enhancement is more significant on scar than on myocardium. This can be explained by the fact that scar has more irregular shape, smaller area and visually challenging pixels which may result in higher uncertainty compared to myocardium (Fig. 2 (b)).

The apical and basal slices of the left ventricle are more difficult to segment than mid-ventricular images even for human experts [3,18]. Particularly at the apical slices, the MRI resolution is very low that it is even difficult to resolve size of small structures (first row in Fig. 3). Assessing the segmentation performance and uncertainties at different slice positions of the left ventricle, it can be observed that the apical slices have the highest epistemic uncertainty (lowest DiceWithinSamples) among the slices (Fig. 2 (b)). Similarly, in the comparison of segmentation performance, most of the improvements due to the addition of uncertainty information (proposed method) are predominantly on the apical slices (Fig. 2 (a)). The DSC increased by $2 \%$ for scar and by almost $1 \%$ for myocardium in the apical slices. While the segmentation performance of the proposed method at the mid and basal slices are similar or slightly better than the baseline method. This tells us that the addition of uncertainty information to the loss function is more advantageous to the semantically and visually challenging images which generate higher epistemic uncertainty. This confirms our initial assumption about the proposed method.

Table 1. Comparison of myocardium and scar (infarction) segmentation performance of the baseline method and the proposed method in terms of geometrical and clinical metrics obtained on the test set (50 cases). The values mentioned are mean (standard deviation). The best results are in bold. VD is the volume error. For DSC, the higher the value the better whereas for $\mathrm{HD}$, Brier score (BS) and VD the lower is the better.

\begin{tabular}{|l|l|l|l|l|l|l|}
\hline \multirow{2}{*}{ Method } & \multicolumn{3}{|c|}{ Myocardium } & \multicolumn{3}{c|}{ Infarction } \\
\cline { 2 - 7 } & DSC (\%) & HD (mm) & $\begin{array}{l}\text { BS } \\
\left(10^{-2}\right)\end{array}$ & DSC $(\%)$ & VD $\left(\mathrm{cm}^{3}\right)$ & $\begin{array}{l}\text { BS } \\
\left(10^{-2}\right)\end{array}$ \\
\hline Baseline & $88.0(2.63)$ & $12.1(7.79)$ & $4.03(2.45)$ & $65.0(29.7)$ & $3.04(5.0)$ & $1.19(1.81)$ \\
\hline Proposed & $\mathbf{8 8 . 2 ( 2 . 5 5 )}$ & $\mathbf{1 1 . 8 ( 7 . 2 6 )}$ & $\mathbf{3 . 8 6 ( 2 . 8 )}$ & $\mathbf{6 7 . 6 ( 2 8 . 8 )}$ & $\mathbf{2 . 9 9 ( 4 . 5 5 )}$ & $\mathbf{1 . 1 8 ( 1 . 8 3 )}$ \\
\hline
\end{tabular}

Fig. 3 shows examples of the segmentation results of baseline and proposed method at apical, mid-ventricular and basal slices. At the apical slice, one can see that the segmentation result of the baseline method has a lot of errors. In the generated uncertainties (sample variance), the incorrectly segmented regions have higher uncertainty. The proposed method, which utilizes the sample variance as part of the loss, minimized the segmentation errors of the baseline. Similarly, our proposed method produced more robust segmentation results at the mid and basal slices. From the results, we can say that the uncertainty captures relevant information that can be leveraged to improve the segmentation result. 

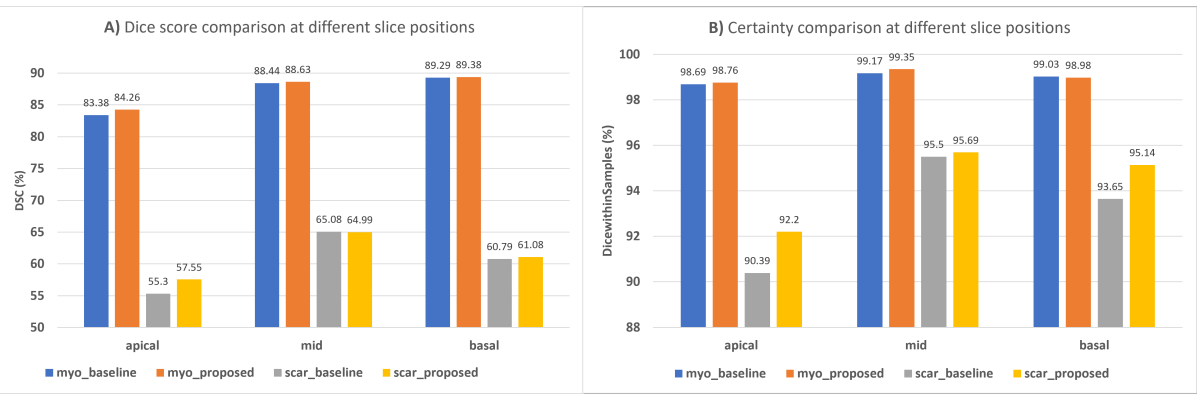

Fig. 2. Dice score (A) and certainty (B) comparison of the baseline and proposed method at different slice locations. Myo_baseline and Scar_baseline refer to myocardium and scar Dice score or certainty of the baseline method respectively. Similarly, Myo_proposed and Scar_proposed refer to myocardium and scar Dice score or certainty of the proposed method.

Regarding the probability calibration, the proposed method produced more calibrated probabilities than the baseline method on both myocardium and scar as it yielded lower Brier score. This suggests that using MC-dropout during training and the addition of uncertainty information to the loss can improve not only the segmentation accuracy but also the calibration of the probabilities.

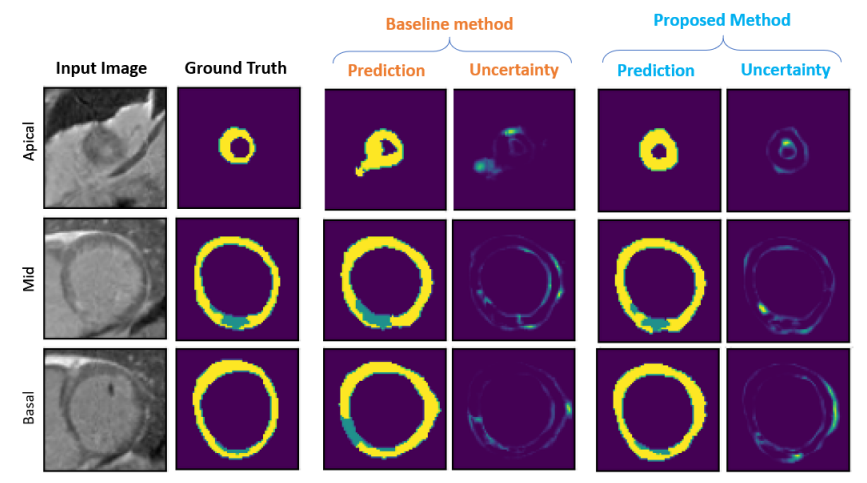

Fig. 3. Qualitative results comparison of the proposed method with the baseline on a typical cardiac MRI. The generated uncertainty is sample variance. Scar (green) and myocardium (yellow).

\subsection{Comparison with state of the art}

Table 2 shows the comparison of the proposed method with state of the art methods on EMIDEC challenge. One can observe that the proposed method 
outperformed the state of the art methods on most of the geometrical and clinical metrics. Our proposed method yielded much better results in all metrics than Feng et al. [5], which used a dilated 2D Unet. Zhang [23] and Ma [14] employed nnU-Net based segmentation pipeline which is similar to the proposed method's pipeline. However, the proposed method, which utilizes a novel loss function that took into account the uncertainty generated during training, outperformed these two top ranked methods. In the segmentation of infarction, the proposed method reduced the average volume error from $3.12 \mathrm{~cm}^{3}$ to $2.99 \mathrm{~cm}^{3}$ and the percentage from $2.38 \%$ to $2.29 \%$ compared to Zhang's [23] method. In terms of the Dice score of infarction, Zhang's [23] method achieved better results, however, this was obtained using two stage cascaded framework which is more computationally expensive framework.

Table 2. Comparison of segmentation performance with state of the art methods on EMIDEC challenge's test set (50 cases). Bold results are the best.

\begin{tabular}{|l|c|c|l|l|l|l|l|l|l|}
\hline \multirow{2}{*}{ Authors } & \multicolumn{3}{|c|}{ Myocardium } & \multicolumn{3}{c|}{ Infarction } & \multicolumn{3}{c|}{ NoReflow } \\
\cline { 2 - 10 } & $\begin{array}{l}\text { DSC } \\
(\%)\end{array}$ & $\begin{array}{c}\text { VD } \\
\left(\mathrm{cm}^{3}\right)\end{array}$ & $\begin{array}{c}\text { HD } \\
(\mathrm{mm})\end{array}$ & $\mathbf{D S C}(\%)$ & $\mathbf{V D}\left(\mathrm{cm}^{3}\right)$ & $\mathbf{P D}(\%)$ & $\mathbf{D S C}(\%)$ & $\mathbf{V D}\left(\mathrm{cm}^{3}\right)$ & $\mathbf{P D}(\%)$ \\
\hline Zhang & 87.86 & 9.26 & 13.01 & $\mathbf{7 1 . 2 4}$ & 3.12 & 2.38 & 78.51 & 0.635 & 0.38 \\
\hline Ma & 86.28 & 10.2 & 14.31 & 62.24 & 4.87 & 3.50 & 77.76 & 0.830 & 0.49 \\
\hline Feng et al. & 83.56 & 15.2 & 33.77 & 54.68 & 3.97 & 2.89 & 72.22 & 0.883 & 0.53 \\
\hline Proposed & $\mathbf{8 8 . 2 2}$ & $\mathbf{9 . 2 3}$ & $\mathbf{1 1 . 7 8}$ & 67.64 & $\mathbf{2 . 9 9}$ & $\mathbf{2 . 2 9}$ & $\mathbf{8 1 . 0 0}$ & $\mathbf{0 . 6 0 1}$ & $\mathbf{0 . 3 7}$ \\
\hline
\end{tabular}

\section{Conclusion}

In this paper, we proposed a segmentation model that generates uncertainty estimates during training using MC-dropout method and utilizes the uncertainty information to enhance the segmentation results by incorporating it into the loss function. The proposed method was evaluated on the publicly available EMIDEC dataset. It achieved state of the art results outperforming the top ranked methods. Assessing the segmentation performance of the proposed method at different slice positions, we observed that the Dice scores of the more challenging apical slices increased much more than the other slice positions. Furthermore, the improvements in the more difficult scar segmentation was higher than that of myocardium segmentation. In the quantitative and qualitative results, we demonstrated that the uncertainty information was indeed advantageous in enhancing the segmentation performance and the improvements were more significant at the semantically and visually challenging images which have higher epistemic uncertainty. In addition, the proposed method produced more calibrated segmentation probabilities.

The main limitation of our method is that it takes more time to train than the baseline method as it uses MC-dropout during training to generate the uncertainty estimates. However, once it is trained, the inference time is exactly the 
same as the baseline method. Future work will focus on utilizing the uncertainty estimates generated by other Bayesian methods such as variational inference to improve the segmentation performance. We will also extend the evaluation onto other challenging public datasets.

\section{Acknowledgements}

This work was supported by the French National Research Agency (ANR), with reference ANR-19-CE45-0001-01-ACCECIT. Calculations were performed using HPC resources from DNUM CCUB (Centre de Calcul de l'Université de Bourgogne). We also thank the Mesocentre of Franche-Comté for the computing facilities.

\section{References}

1. Abbas, A., Matthews, G.H., Brown, I.W., Shambrook, J., Peebles, C., Harden, S.: Cardiac MR assessment of microvascular obstruction. The British journal of radiology $88 \mathbf{1 0 4 7}, 20140470$ (2015)

2. Arega, T.W., Bricq, S.: Automatic Myocardial Scar Segmentation from Multisequence Cardiac MRI Using Fully Convolutional Densenet with Inception and Squeeze-Excitation Module. In: Myocardial Pathology Segmentation Combining Multi-Sequence CMR Challenge. pp. 102-117. Springer (2020)

3. Bernard, O., Lalande, A., Zotti, C., Cervenansky, F., Yang, X., Heng, P., Cetin, I., Lekadir, K., Camara, O., Ballester, M.G.G., Sanromá, G., Napel, S., Petersen, S., Tziritas, G., Grinias, E., Khened, M., Kollerathu, V., Krishnamurthi, G., Rohé, M.M., Pennec, X., Sermesant, M., Isensee, F., Jäger, P.F., Maier-Hein, K., Full, P.M., Wolf, I., Engelhardt, S., Baumgartner, C.F., Koch, L.M., Wolterink, J., Isgum, I., Jang, Y., Hong, Y., Patravali, J., Jain, S., Humbert, O., Jodoin, P.M.: Deep Learning Techniques for Automatic MRI Cardiac Multi-Structures Segmentation and Diagnosis: Is the Problem Solved? IEEE Transactions on Medical Imaging 37, 2514-2525 (2018)

4. Blundell, C., Cornebise, J., Kavukcuoglu, K., Wierstra, D.: Weight uncertainty in neural network. In: International Conference on Machine Learning. pp. 1613-1622. PMLR (2015)

5. Feng, X., Kramer, C., Salerno, M., Meyer, C.H.: Automatic Scar Segmentation from DE-MRI Using 2D Dilated UNet with Rotation-Based Augmentation. In: M\&Ms and EMIDEC/STACOM@MICCAI (2020)

6. Fortunato, M., Blundell, C., Vinyals, O.: Bayesian recurrent neural networks. arXiv preprint arXiv:1704.02798 (2017)

7. Gal, Y., Ghahramani, Z.: Dropout as a Bayesian approximation: Representing model uncertainty in deep learning. In: international conference on machine learning. pp. 1050-1059. PMLR (2016)

8. Girum, K.B., Skandarani, Y., Hussain, R., Grayeli, A.B., Créhange, G., Lalande, A.: Automatic Myocardial Infarction Evaluation from DelayedEnhancement Cardiac MRI using Deep Convolutional Networks. In: M\&Ms and EMIDEC/STACOM@MICCAI (2020) 
9. Isensee, F., Jaeger, P.F., Kohl, S.A., Petersen, J., Maier-Hein, K.H.: nnU-Net: a self-configuring method for deep learning-based biomedical image segmentation. Nature methods 18(2), 203-211 (2021)

10. Jungo, A., Reyes, M.: Assessing reliability and challenges of uncertainty estimations for medical image segmentation. In: International Conference on Medical Image Computing and Computer-Assisted Intervention. pp. 48-56. Springer (2019)

11. Kate Meier, C., Oyama, M.A.: Chapter 41 - Myocardial Infarction. In: Silverstein, D.C., Hopper, K. (eds.) Small Animal Critical Care Medicine, pp. 174-176. W.B. Saunders, Saint Louis (2009). https://doi.org/https://doi.org/10.1016/B9781-4160-2591-7.10041-4, https://www.sciencedirect.com/science/article/pii/ B9781416025917100414

12. Kendall, A., Badrinarayanan, V., Cipolla, R.: Bayesian segnet: Model uncertainty in deep convolutional encoder-decoder architectures for scene understanding. arXiv preprint arXiv:1511.02680 (2015)

13. Lalande, A., Chen, Z., Decourselle, T., Qayyum, A., Pommier, T., Lorgis, L., de la Rosa, E., Cochet, A., Cottin, Y., Ginhac, D., et al.: Emidec: A database usable for the automatic evaluation of myocardial infarction from delayed-enhancement cardiac MRI. Data 5(4), 89 (2020)

14. Ma, J.: Cascaded Framework for Automatic Evaluation of Myocardial Infarction from Delayed-Enhancement Cardiac MRI. arXiv preprint arXiv:2012.14556 (2020)

15. Mehrtash, A., Wells, W.M., Tempany, C.M., Abolmaesumi, P., Kapur, T.: Confidence calibration and predictive uncertainty estimation for deep medical image segmentation. IEEE transactions on medical imaging 39(12), 3868-3878 (2020)

16. Nair, T., Precup, D., Arnold, D.L., Arbel, T.: Exploring uncertainty measures in deep networks for multiple sclerosis lesion detection and segmentation. Medical image analysis 59, 101557 (2020)

17. Ng, M., Guo, F., Biswas, L., Petersen, S.E., Piechnik, S.K., Neubauer, S., Wright, G.: Estimating Uncertainty in Neural Networks for Cardiac MRI Segmentation: A Benchmark Study. arXiv preprint arXiv:2012.15772 (2020)

18. Petitjean, C., Dacher, J.N.: A review of segmentation methods in short axis cardiac MR images. Medical image analysis 15(2), 169-184 (2011)

19. Roy, A.G., Conjeti, S., Navab, N., Wachinger, C.: Inherent brain segmentation quality control from fully convnet Monte Carlo sampling. In: International Conference on Medical Image Computing and Computer-Assisted Intervention. pp. 664-672. Springer (2018)

20. Sander, J., de Vos, B.D., Wolterink, J.M., Išgum, I.: Towards increased trustworthiness of deep learning segmentation methods on cardiac MRI. In: Medical Imaging 2019: Image Processing. vol. 10949, p. 1094919. International Society for Optics and Photonics (2019)

21. Wang, G., Li, W., Ourselin, S., Vercauteren, T.: Automatic brain tumor segmentation based on cascaded convolutional neural networks with uncertainty estimation. Frontiers in computational neuroscience 13, 56 (2019)

22. Zabihollahy, F., White, J.A., Ukwatta, E.: Myocardial scar segmentation from magnetic resonance images using convolutional neural network. In: Medical Imaging 2018: Computer-Aided Diagnosis. vol. 10575, p. 105752Z. International Society for Optics and Photonics (2018)

23. Zhang, Y.: Cascaded Convolutional Neural Network for Automatic Myocardial Infarction Segmentation from Delayed-Enhancement Cardiac MRI. arXiv preprint arXiv:2012.14128 (2020) 